\title{
A Longitudinal Analysis of Environment and Risk of Obesity in the US
}

\author{
Yanqing $\mathrm{Xu}^{1}{ }^{*}$, Yanhong $\mathrm{Liu}^{2}$, Tian Chen ${ }^{3}$, Wanyun Shao ${ }^{4}$, Zhongliang $\mathrm{Fu}^{5}$ \\ ${ }^{1}$ Department of Geography and Planning, University of Toledo, Toledo, USA \\ ${ }^{2}$ School of Intervention and Wellness, University of Toledo, Toledo, USA \\ ${ }^{3}$ Department of Mathematics \& Statistics, University of Toledo, Toledo, USA \\ ${ }^{4}$ Department of Sociology, Anthropology and Geography, Auburn University at Montgomery, Montgomery, USA \\ ${ }^{5}$ School of Remote Sensing and Information Engineering, Wuhan University, Wuhan, China \\ Email: *yanqing.xu@utoledo.edu
}

How to cite this paper: Xu, Y., Liu, Y.H., Chen, T., Shao, W.Y. and Fu, Z.L. (2017) A Longitudinal Analysis of Environment and Risk of Obesity in the US. Journal of Geoscience and Environment Protection, 5, 204220.

https://doi.org/10.4236/gep.2017.59015

Received: August 15, 2017

Accepted: September 19, 2017

Published: September 22, 2017

Copyright () 2017 by authors and Scientific Research Publishing Inc. This work is licensed under the Creative Commons Attribution International License (CC BY 4.0).

http://creativecommons.org/licenses/by/4.0/

\begin{abstract}
Obesity is a fast-growing global health crisis and the epidemic is about to get worse. Environment has been shown to influence physical activity and people's body weight. Utilizing Centers for Disease Control and Prevention 2004-2010 waves of the continuous obesity data, this study conducted longitudinal analyses to examine neighborhood built environment and obesity risk controlling for the effects of socio-demographic characteristics. This study presents a comprehensive effort to understand the relationship between the environment and physical inactivity and obesity across the entire contiguous US. When constructing measures of the built environment, Geographic Information Systems (GIS) were used to calculate street connectivity, walk score and food environment. In addition to the built environment, the natural environment, which was represented by the annual number of extreme weather events from 2004-2010, was taken into account in explaining variation of physical inactivity and obesity across the contiguous US. Results show that higher street connectivity and walk score are related to lower physical inactivity and obesity rates, while the ratio of fast-food restaurants and number of extreme weather events are positively related to physical inactivity and obesity. The results are believed to provide policy-makers and planners with useful insights into the dynamics between the environment and obesity epidemic. Further, the significant effects of extreme weather invite more studies to investigate the relationship between the natural environment and obesity.
\end{abstract}

\section{Keywords}

Obesity, Physical Inactivity, Built Environment, Longitudinal Study, The US 


\section{Introduction}

Obesity has risen as a global issue in all countries and has been prevalent in both adults and children [1]. The WHO statistics showed that more than 600 million people worldwide were obese; $13 \%$ of adults aged 18 years and above and 41 million children aged 5 years and below were obese in 2014 [2]. A combination of factors may have contributed to obesity including proximal drivers (e.g., human behaviors; physical activity) and those considered as distant drivers such as built environment, food system, policies and other social factors [1].

The relationship between obesity or physical activity and built environment has drawn intense attention in the past decade [e.g., [3] [4] [5]]. From an ecological perspective, human behaviors are influenced by multiple factors, thus changes in built environment are likely to have far reaching impact on people's physical activities [6]. Built environment refers to all human-made places that constitute people's living environment including parks and recreation centers, play grounds, community layout, roads, and transportation system [7]. Built environment factors such as land use, population density, road connectivity, transportation patterns, and food environment have been investigated in relation to physical activity and obesity risk [3] [8] [9].

Evidences consistently support that neighborhood walkability was positively associated with physical activity, namely, residents in highly walkable neighborhoods reported a higher level of physical activity in comparison with those from lowly-walkable neighborhoods [10] [11] [12]. Key components of neighborhood walkability include residential density, land use, and road connectivity [12]. Neighborhood walkability was estimated using Walk Score based on different amenity categories [13]. The current literature also revealed the relationship between the socioeconomic status of a neighborhood and residents' walking for transport. Specifically, residents from low socioeconomic neighborhoods reported more utilization of walking for transport than residents from high socioeconomic neighborhoods who utilized more motor transportation [14].

Food environment was another significant predictor of obesity. The density of food outlets was found significantly associated with the BMI scores of individuals based on a study of 13,102 adults who live in New York City [8]. Rundle et al. [8] also found that a higher density of healthy food outlets was correlated with a lower mean of BMI, whereas a higher density of unhealthy food outlets was associated with a higher level of BMI in individuals. The results were solidified by a 30-year longitudinal study on 3113 residents living in Massachusetts where residential proximity to the closest fast-food restaurant was associated with participants' BMI scores, and an increase in driving distance to the nearest fast-food restaurant was associated with a decrease in participants' BMI scores [15]. Similar findings were found pertaining to the role of built environment in child obesity. A literature review manifested a positive association between more positive built environment (e.g., high walkability or bikeability, high level of land use mix, more accessible destinations) and higher level of child physical activity [16]. 
Additionally, accessibility to fast-food restaurants and convenient stores increases the likelihood of child obesity [16].

Despite the paucity of literature on the link between weather and obesity, previous studies have examined the impact of extreme weather on physical activity. Given that being outdoors is a significant indicator of physical activity [17] [18] [19] [20], extreme weather can pose a barrier for individuals to being outdoors and therefore prevent them from getting engaged in physical activity. Indeed, Tucker and Gilliland [21] conducted a comprehensive literature review on thirty-seven studies from eight countries, and found clear evidence that participation in physical activity is constrained by extreme weather. Physical activity has been identified as a determinant of obesity [22]. It is reasonable to assume that variables identified as factors of physical activity can further cause obesity. A recent empirical study has showed that extreme temperatures represented by hot summers and cold winters can lead to obesity [23]. Hereby, we speculate that more frequent extreme weather events can promote obesity rates in the contiguous US.

However, most studies have been based on cross-sectional analyses and most of the longitudinal studies had only focused on social environments ignoring parameters of the built environment [24] [25]. In addition, the studies based on cross-sectional data involve confounding variables such as walking context and residential self-selection, which may have led to inaccurate findings in regards to the relationship between built environment and physical activity or obesity [26]. Longitudinal analyses are thus desirable to reflect changes in physical activity, obesity, and/or the Body Mass Index (BMI) patterns that are associated with changes that occurred in built environment [27] [28] [29]. The majority of the longitudinal studies had only focused on social parameters of the neighborhood environment without considering characteristics of the built environment and few research examined the longitudinal influence of weather variables. In this study, we seek to explore the longitudinal relationships between sociodemographic environments and physical inactivity/obesity over 7 years, taking into account the neighborhood built environments. Furthermore, the effect of various urbanicity levels and natural environment variable (number of extreme weather events) were examined through the study. The conceptual framework of the study is shown in Figure 1.

\section{Data and Methods}

\subsection{Variables and Data Source}

The study area is 3109 counties in the 48 states and Washington D.C. Alaska and Hawaii are excluded due to their non-contiguity with the conterminous US. Aggregated data for leisure-time physical inactivity prevalence and obesity prevalence were used as dependent variables respectively. The National Health Interview Survey (NHIS) has asked different questions on leisure-time physical activity for purposes of national surveillance [30]. The physical inactivity is the ratio 
Social factors

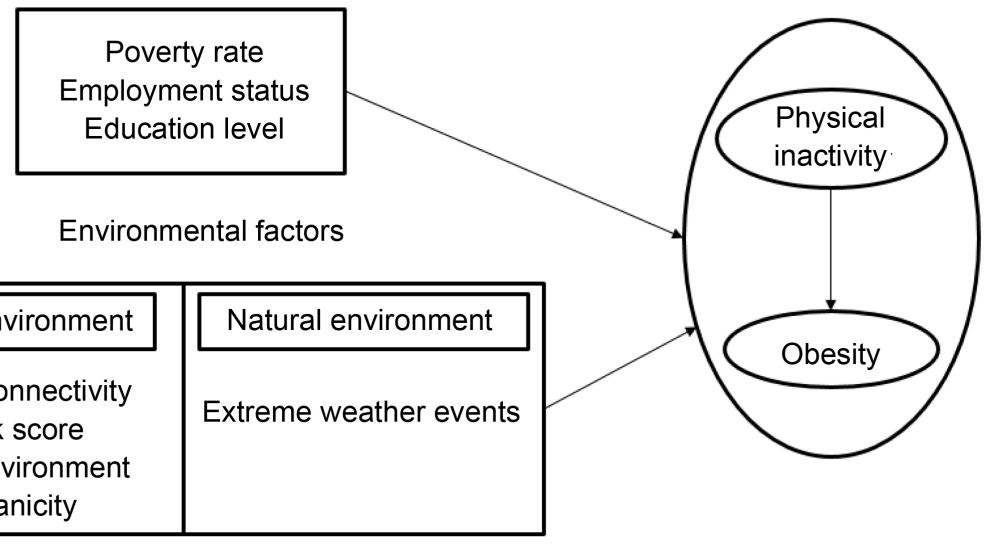

Figure 1. Conceptual framework relating environments to physical inactivity and obesity.

of the population who are physically inactive over the total population in each county. BMI $=$ mass $(\mathrm{kg}) /(\text { height }(\mathrm{m}))^{2}$ was used to measure obesity, if respondents consider their BMI values were over 30 . Obesity rate is calculated as the percentage of the obese population over all population.

These county data indicators can be downloaded from the Centers for Disease Control and Prevention (CDC) website

(https://www.cdc.gov/diabetes/atlas/countydata/atlas.html) from 2004-2010. Data collected in 2011 and forward reflect a change in weighting methodology and combined landline and cell phone respondents, which means that estimates cannot be directly compared with estimates from data collected in 2010 and before [31]. The explanatory variables include four sociodemographic variables: population density (i.e., number of population per square mile), poverty rate, employment status and education level, and three built environment variables: street connectivity, walk score and food environment. Street connectivity and walk score were constructed from GIS-based measures and food environment was measured by the ratio of fast-food restaurants over the total restaurants; the effect of urbanicity according to different levels of urban categories; number of extreme weather events which was used as one measure of natural environments in the study.

\subsubsection{Social Environment}

Population density is measured as the total residents per square mile from the 2010 US Census. The average population density of the 3109 counties is 255.77 , and the Loving County in Texas has the lowest population density while four counties New York, Kings, Bronx and Queens from New York have the highest population density. Poverty data is from the US Census Bureau's Small Area Income and Poverty Estimates (SAIPE) program which provides annual estimates of poverty statistics for all counties [32]. The average poverty rate among all the counties from 2004-2010 is $15.44 \%$. Labor force data was downloaded from local area unemployment statistics provided by Bureau of Labor Statistics 
(https://www.bls.gov/lau/tables.htm). The average unemployment rate is $6.44 \%$ and the rate has increased to more than $9 \%$ in 2009 and 2010. Education data was downloaded from United Stated Department of Agriculture (USDA) Economic Research Service (https://www.ers.usda.gov/data-products/county-level-data-sets.aspx). We couldn't get the annual education data, since the 2000 data is from US. Census Bureau and the decennial Census of population while the data for 2010-2014 is a 5-year average from the Census Bureau's American Community Survey [33]. Education data includes the percentages of adults with less than a high school diploma, with a high school diploma only, completing some college or associate's degree and a bachelor's degree or higher, respectively. We used the percent of adults with less than a high school diploma (express as "percentage of less than high school" in the following contents) in this study.

\subsubsection{Built Environment}

\section{1) Street connectivity}

Street connectivity was defined as the number of intersections per square mile in an area or along a certain street network. Although our study unit is county level, we calculated the index of street connectivity for each census tract and then aggregated to county adjusted by population. The conventional measure of street connectivity directly from a large geographic area such as a county can be biased with the majority of residents concentrated in a limited urban area [34]. Road network was constructed from StreetMap North America included with ArcGIS Desktop. The Environmental System Research Institute (ESRI) is authorized by TomTom (TeleAtlas) to use their four-year-old streets database, updating every two years. The TomTom streets product varies in street segment count depending on the processing they have done to merge adjacent street segments with common attributes in any given year [35]. We calculated the street connectivity for 2004-2006 by using the data CD-ROMs distributed with ArcGIS 10.0 and 2007-2010 with ArcGIS 10.2.

\section{2) Walk score}

Walk score is kept the same through 2004-2010 since we cannot get the past real-time data. We first obtained the walk score in census tracts, and then aggregated to the county level by using the Walk Score API [36]. The aggregation takes population as the weight term such as

$$
w_{k}=\sum_{i=0}^{n_{k}} p_{i} * \frac{w_{i}}{p_{k}}
$$

where $w_{k}$ is the walk score in county $k, n_{k}$ is the number of census tract units in county $k, p_{i}$ is the population of census tract $i$ within county $k$, and $p_{k}$ is the total population in county $k$. Walk Score API accepts the latitude and longitude of the requested location or a specific address. For each point, Walk Score analyzes hundreds of walking routes to nearby amenities, such as schools, parks, restaurants, and grocery stores. Data source include Google, Education.com, Open 
Street Map, the US Census, Localeze and used-defined places [37]. We used the population-weighted centroids of the census tracts from census block 2010 data as input parameters, instead of simple geographic centroid, to avid bias caused by population concentration in a limited space within a large area [38] [39]. We developed a Python program to automatically request walk scores from the server through the Walk Score API. Walk score ranges from 0 (car-dependent) to 100 (walker's paradise).

\section{3) Food environment}

Besides lacking of physical activity, eating unhealthy is another important factor related to obesity. There are many ways to measure food environment: Xu et al. [40] used fast-food restaurant accessibility to measure food environment at the zip code level; some studies used the number of fast-food restaurants per capita to measure food environment [41] [42]. In our research, we used the ratio of fast-food restaurants to all restaurants (fast-food and full-service restaurants) numbers to measure food environment, which can account for the availability of options between healthy and unhealthy food by consumers. The data is from the County Business Patterns (CBP), an annual series that provide subnational economic data by industry

(http://www.census.gov/programs-surveys/cbp/data/datasets.html).

\subsubsection{Urbanicity}

According to Lopez and Hyness [43], inner city residents usually have higher population density, greater street connectivity and higher walk score, but yet they still have higher physical inactivity and obesity rates when compared to people living in suburban areas. In the meantime, inner city neighborhood tend to have more disadvantaged population (i.e., minorities, low socioeconomic status) with less attractive and safe environments for physical activity [44]. Finer classifications and more reasonable measures of urbanicity are necessary to improve research on the urbanicity-health relationships than the simple classification of urban and rural regions. In this research, we used the 2006 National Center for Health Statistics (NCHS) for 2004-2006 and 2013 NCHS for 2007-2010. 2006 NCHS is based on the Office of Management and Budget's (OMB) December 2005 delineation of Metropolitan/Micropolitan Statistical Areas (MSA) and Vintage 2004 postcensal estimates of the resident US population, while 2013 NCHS is based on OMB February 2013 MSA and Vintage 2012 [45]. Both classifications in 2006 and 2013 contain six levels of urbanicity categories, including large central metro, large fringe metro, medium metro, small metro, micropolitan and nor-core [46]. There are only small differences between the classifications of these two years.

\subsubsection{Extreme Weather Events}

The extreme weather events data comes from National Weather Service (NWS) at National Climatic Data Center (NCDC). The data records "the occurrence of storms and other significant weather phenomena having sufficient intensity to 
cause loss of life, injuries, significant property damage, and/or disruption to commerce, and it also includes weather phenomena that "generate media attention" and "other significant meteorological events such as maximum or minimum temperatures or precipitation" (NWS 10-1605, 4). The data has 48 types of extreme weather events. We construct the annual number of extreme weather events per county from 2004-2010 based on this data.

\subsection{Analytical Strategy}

Generalized Estimating Equations (GEE) was employed to address the dependency of repeated observations of obesity rate over years in the same county. We opted for the GEE rather than a parametric alternative such as the generalized linear mixed-effects model, because of robustness of GEE estimates for a wider class of data distributions. Physical inactivity rate and obesity rate at 2004 were treated as the baseline level and introduced into the GEE model as a control variable respectively. Another control variable added was the population density. The model also included variables of interest to assess their effects on obesity rate: poverty rate, percent of adults with lower than a high school diploma, unemployment rate, street connectivity, walk score, the ratio of fast-food restaurants, urbanicity level and annual number of extreme weather events. Due to extremely skewered distribution of walk score, observations were dichotomized into either low $(<25)$ or high $(\geq 25)$ groups and around $80 \%$ fell in the low group. All statistical tests were two-sided; $p$-values of $<0.05$ were considered to be statistically significant. All statistical analyses were performed using R 3.2.2.

\section{Results}

Before proceeding to the findings, we first present patterns on the rates of physical inactivity/obesity and descriptive statistics of the county level variables. In Figure 2, we show the annual trend in physical inactivity and obesity rates from 2004 to 2010. The mean value of obesity rate has increased year by year, from $25.20 \%$ in 2004 to $30.48 \%$ in 2010 , while the mean value of physical inactivity rate presents almost the same pattern with a slight difference. The mean rate is a slightly lower in 2005 than in 2004, and slightly lower in 2010 than in 2009. In order to save space, we showed the distribution patterns of all the variables in
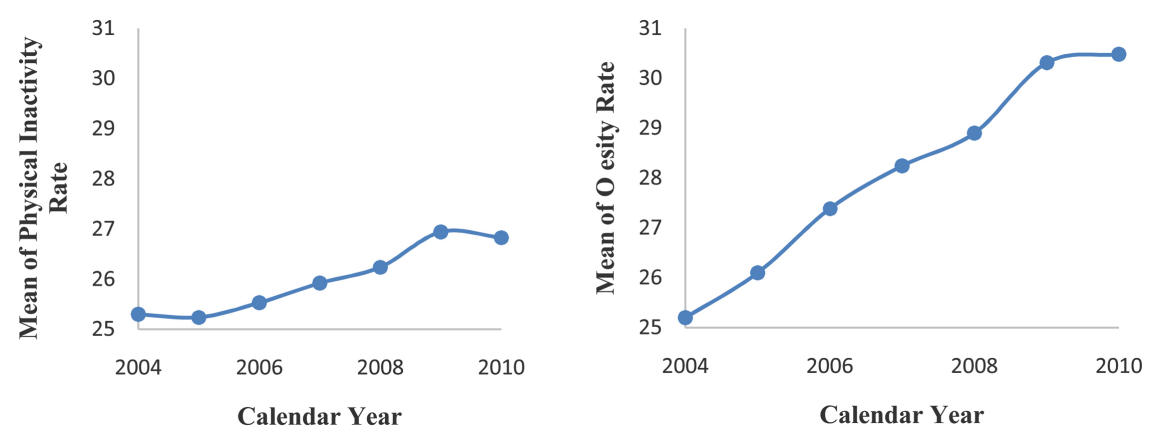

Figure 2. Physical inactivity and obesity rates in the United States, 2004 to 2010. 
2010 in Figure 3 and Figure 4. From Figure 3(a) and Figure 3(b), we can see that higher overall obesity and physical inactivity are clustered in the south-east central region of the US Greene County, Alabama, has the highest obesity rate

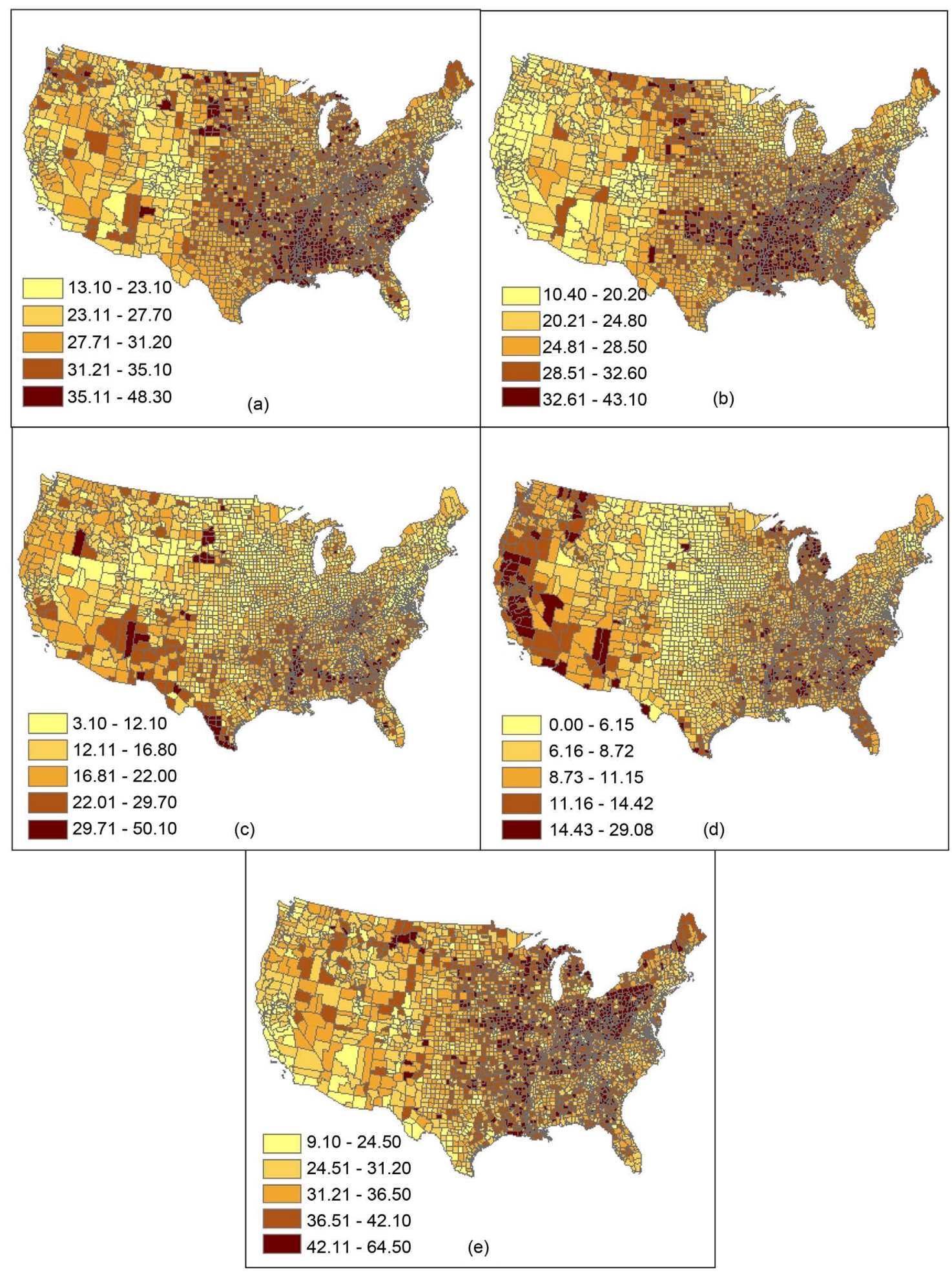

Figure 3. Distribution patterns of variables in 2010: (a) Obesity rate; (b) Physical inactivity rate; (c) Poverty rate; (d) Unemployment rate; (e) Percentage of less than high school. 


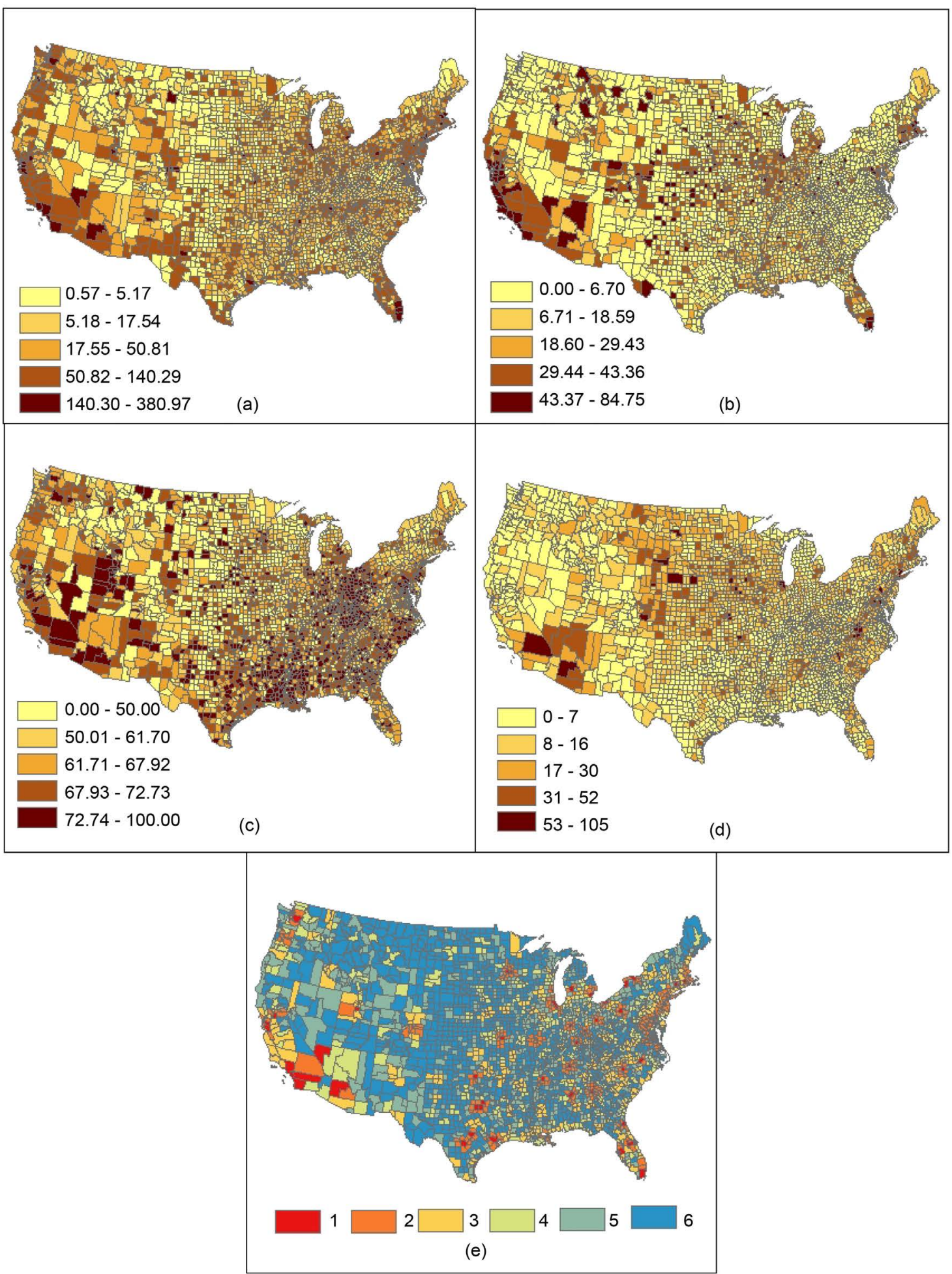

Figure 4. Distribution patterns of variables in 2010: (a) Street connectivity; (b) Walk score; (c) Food environment; (d) Extreme weather events; (e) Urbanicity.

at $48.3 \%$, while Wyoming County, West Virginia has the highest physical inactivity rate at $43.1 \%$. The average poverty rate, unemployment rate and percentage of adults with lower than a high school diploma in 2010 are $16.79 \%, 9.36 \%$ 
and $15.49 \%$ individually. From Table 1, the five aforementioned variables showed significant difference between baseline and follow-up results according to the Mann-Whitney U test.

From Figure 4(a) and Figure 4(b), street connectivity and walk score show high values along the West Coast and the urbanized northeast of the US San Francisco in California, District of Columbia, Baltimore City in Maryland and Suffolk County in Massachusetts have extremely high values of street connectivity and walk score. The ratio of fast-food restaurants to all restaurants does not show many obvious patterns from Figure 4(c). Ohio, Louisiana and Maryland have the highest average ratio of fast-food restaurants and South Dakota, North Dakota and Nebraska have the lowest average ratio. In 2010, there were 105 extreme weather events in Cook County, Illinois and 102 in Polk County, Iowa. Iowa and Texas has the highest total number of extreme weather events in this year. Figure 4 (e) shows the distribution of urban-rural categories, in which the warm colors 1, 2, 3 and 4 stand for metro areas (large central, large fringe, medium and small) and the cold colors 5 and 6 stand for non-metro areas (micropolitan and rural). Among the 3109 counties, 1086 (approximately 35\%) are metro areas while the rest are non-metro areas.

Table 2 represents the regression coefficients and standard error from GEE-analysis, investigating the longitudinal relationship between physical inactivity/obesity (outcome variable) and neighborhood socio and built environment over 7 years. Generalized estimating equations were used to estimate the coefficients for physical inactivity and obesity, respectively. GEE takes into account

Table 1. Characteristics of the variables.

\begin{tabular}{|c|c|c|}
\hline & Baseline & Follow-Up \\
\hline Obesity rate & $25.20(18.75,31.65)$ & $28.57(20.43,36.72)$ \\
\hline $\begin{array}{c}\text { Physical inactivity } \\
\text { rate }\end{array}$ & $25.30(15.20,35.40)$ & $26.12(16.16,36.08)$ \\
\hline Poverty rate & $13.77(3.69,23.85)$ & $15.72(3.33,28.10)$ \\
\hline $\begin{array}{l}\text { Unemployment } \\
\text { rate }\end{array}$ & $5.66(2.17,9.15)$ & $6.57(0.62,12.51)$ \\
\hline $\begin{array}{l}\text { Less than high } \\
\text { school } \%\end{array}$ & $22.66(5.54,39.78)$ & $20.27(2.94,37.60)$ \\
\hline Street connectivity & $30.26(0,92.72)$ & $32.90(0,108.10)$ \\
\hline $\begin{array}{c}\text { Walking score } \\
(\text { low/high })\end{array}$ & \multicolumn{2}{|c|}{ Low: $79.7 \%$ High: $20.3 \%$} \\
\hline $\begin{array}{l}\text { Fast-food } \\
\text { restaurant ratio }\end{array}$ & $61.1(0.26,0.96)$ & $61.3(0.27,0.96)$ \\
\hline Urbanicity & $2.03 / 11.39 / 10.58 / 10.94 / 22.13 / 42.94$ & $2.19 / 11.84 / 11.90 / 11.42 / 20.49 / 42.17$ \\
\hline $\begin{array}{c}\text { Extreme weather } \\
\text { events }\end{array}$ & $11.29(0,30.85)$ & $12.62(0,35.19)$ \\
\hline
\end{tabular}

Note: Results are presented as 95\% confidence interval. Marked bold: significant difference between baseline and follow-up (Mann-Whitney $U$ test; $\mathrm{p}<0.001)$. 
Table 2. GEE predicting physical inactivity and obesity by US County, 2004 to 2010 .

\begin{tabular}{|c|c|c|c|c|}
\hline & \multicolumn{2}{|c|}{ Physical inactivity } & \multicolumn{2}{|c|}{ Obesity } \\
\hline & Coefficient & $S E$ & Coefficient & $S E$ \\
\hline Intercept & $-1.310^{* * *}$ & 0.266 & 0.325 & 0.176 \\
\hline Baseline & $0.790^{\star * *}$ & 0.009 & $0.942^{\star * *}$ & 0.006 \\
\hline \multicolumn{5}{|l|}{ Socio-demographic variables } \\
\hline Population density & $1.30 \mathrm{e}-4^{\star * *}$ & $2.27 \mathrm{e}-5$ & $-3.60 e-6$ & $7.14 \mathrm{e}-6$ \\
\hline Poverty rate & $0.085^{* * *}$ & 0.007 & $0.022^{* * *}$ & 0.004 \\
\hline Less high school \% & $0.167^{* * *}$ & 0.004 & $0.020^{* * *}$ & 0.002 \\
\hline Unemployment rate & $-0.115^{\star * *}$ & 0.011 & $0.026^{* * *}$ & 0.007 \\
\hline \multicolumn{5}{|l|}{ Built environment variables } \\
\hline Street connectivity & $-0.011^{\star * *}$ & 0.001 & $-0.007^{\star * *}$ & 0.001 \\
\hline Walk score & $-0.451^{\star \star \star}$ & 0.060 & $-0.123^{\star * \star}$ & 0.037 \\
\hline Fast food ratio & $1.43 \mathrm{e}-2^{* * *}$ & 0.151 & $4.84 \mathrm{e}-3^{* * *}$ & 0.087 \\
\hline \multicolumn{5}{|l|}{ Urban-rural classification } \\
\hline \multicolumn{5}{|l|}{$\begin{array}{l}\text { Large central metro } \\
\quad(\text { reference })\end{array}$} \\
\hline Large fringe metro & $0.771^{\star * *}$ & 0.176 & $0.298^{* *}$ & 0.106 \\
\hline Medium metro & 0.071 & 0.177 & $0.240^{*}$ & 0.106 \\
\hline Small metro & 0.039 & 0.182 & $0.390^{* * *}$ & 0.110 \\
\hline Micropolitan & 0.229 & 0.180 & $0.316^{* * *}$ & 0.109 \\
\hline Non-core & 0.283 & 0.184 & 0.211 & 0.111 \\
\hline \multicolumn{5}{|l|}{ Weather variables } \\
\hline Extreme weather events & $0.023^{* * *}$ & 0.002 & $0.010^{\star * *}$ & 0.001 \\
\hline Year (2004 to 2010) & $0.725^{\star * *}$ & 0.018 & $0.902^{\star * *}$ & 0.010 \\
\hline
\end{tabular}

Note. $\mathrm{SE}=$ standard error; $\mathrm{e}-1=10^{-1} .{ }^{*} \mathrm{p}<0.05,{ }^{* *} \mathrm{p}<0.01,{ }^{* *} \mathrm{p}<0.001$.

the dependency of repeated observations from the same subject by specifying a "working correlation structure" and it doesn't require any distribution assumption compared with another alternative, linear mixed effect model. The physical inactivity rate is expected to increase by $0.085 \%$ for each percent increase in poverty rate after adjusting for other variables, while obesity rate is expected to increase by $0.022 \%$. Population density is positively related to physical inactivity but has no effect on obesity. Percent of adults without a high school diploma is expected to be related to an increase in physical inactivity and obesity rates. There is a contradictory finding on unemployment rate: it is positively related to obesity rate while negatively related to physical inactivity rate. The three built environment variables show the same direction of effect on both physical inactivity and obesity. Higher street connectivity and walk score are expected to lower physical inactivity and obesity rates; the ratio of fast-food restaurants is positively related to the two outcome variables. Number of extreme weather events has the same effect as food environment. By comparing with large central metro, people living in large fringe metro is expected to have significant higher 
physical inactivity and obesity rates, while medium metro, small metro and micropolitan have significant higher rate for obesity only.

\section{Discussion}

The study examines several environmental features, including social, built and natural environments in their association with physical inactivity and obesity at the geographic aggregation level, considering time effect from 2004-2010. Street connectivity and walk score confirms the previous findings that walkability play an important role in reducing physical inactivity and obesity [47] [48]. GIS-based measures of walkability have demonstrated a feasible and effective way in the study of public health. Food environment was also measured in a reasonable way to examine its effect on obesity. We considered not only the fast-food restaurants but also the full-service restaurants according to different consumer needs. Furthermore, NCHS six urban-rural categories were added in the model to measure the influences of different geographic settings on physical inactivity and obesity. Besides the commonly used social and built environments, we added one natural environment in this research. The weather variable, annual number of extreme weather events, was used to measure the natural environment that may lead to physical inactivity and thus increase obesity risks.

As to social environment variables, the results for population density are different for physical inactivity and obesity. It is not related to obesity rate but linked to greater physical inactivity risk. Higher population density may suggest better walkability, however, higher population density may be linked to more active transportation, which can result in higher risk of physical inactivity. The relationship between population density and obesity can vary depending on other factors being controlled and needs to be further explored. Higher poverty rate and lower education level are linked to higher risks of physical inactivity and obesity. People with high income and high education are more likely to buy healthy food. Moreover, they are more likely to be aware of health-related information and be more motivated and equipped to follow suggested healthy lifestyles. The inconsistency between the employment status on physical inactivity and obesity suggests possible gaps in data reliability of self-reported physical inactivity and more objectivity body weight and height.

Street connectivity and walk score are objective measures of walkability, which also capture the patterns of mixed land use. The negative relationship of these variables with physical inactivity and obesity rates provides a strong argument for the concept that pedestrian-friendly programming and activity-promoting facilities are related to lower risks of physical inactivity and obesity. While walkability promotes physical activity, food environment is hypothesized to affect dietary intake which is another key energy balance factor. Table 2 shows that fast-food restaurant ratio is strongly positively related to the risk of physical inactivity and obesity $(\mathrm{p}<0.001)$. According to our general life experience, fast-food restaurants are the main source of unhealthy, processed and energy 
dense foods, whereas full-service restaurants provide more healthy food. On the other hand, food price in fast-food restaurants is much cheaper than full-service ones, therefore, people are more likely to intake excessive energy. Our results provide evidence at the national level, rather than a local setting, on the link between built environment and physical inactivity/obesity.

Different urban-rural classifications have different effects on the two outcome variables. From Table 2, large fringe metro is the only classification that has statistical significant higher physical inactivity rate compared with large central metro (reference level). Small metro and micropolitan areas are strongly positively related to obesity risk, while large fringe and medium metro has somewhat weaker but still significantly positive effect. People living in urban areas have more chances to access facilities and more walkable destinations. The relationship between urbanicity and physical inactivity/obesity needs to be further explored. The number of extreme weather events was added as a natural environment. Extreme weather events constitute a hostile environment that discourages people from engaging in physical activities, thus further induce obesity. In other words, if there are more extreme weather conditions, people are more likely to stay inside, which will reduce their chances to participate in physical activities and increase the likelihood of eating more while staying at home. We will add more variables to measure the nature environment in future studies of physical activity and obesity.

\section{Conclusions}

The research agenda on revealing the obesity-environment is far from being complete. Several limitations of this study need to be acknowledged. First, although we have included various variables retrieved from the literature, there are still some variables that need to be considered. For example, the land use mix, other weather variables (temperature, precipitation etc.), and crime rate in the neighborhood. Second, the self-report surveys may not be the most reliable measures of physical activity. Future studies need to account for these biases and adopt a comprehensive measure to capture individual physical activity. Furthermore, the impacts of unemployment status on physical inactivity and obesity need to be further explored, given the seemingly contradictory results in the present study. Lastly, we used aggregated data in this study ignoring individual variability. Individual behaviors such as eating habit and physical activity do not occur itself; rather, they are influenced by socio-environmental factors including built and natural environment. We will include individual-level risk factors in the future to overcome the possible ecological fallacy, where relationships observed in groups are assumed to hold for individuals.

In conclusion, the key purpose of this research was to explore the role of neighborhood social characteristics, and built and natural environment attributes in contributing to physical inactivity and obesity risk over a span of seven years across the contiguous US. A space-time framework was used in this study to 
analyze different years, instead of one single year which could predict the spatio-temporal pattern of the obesity-environment relationship. Through comprehensive data preparation and analyses, we made several important findings: higher poverty rate, lower education level, lower walkability, higher ratio of fastfood restaurants, larger number of extreme weather events are related to higher risks of physical inactivity and obesity, and different urban-rural levels have different effects on these risks. Furthermore, the obesity problem and related environment factors for counties in different years were analyzed using GEE model with a GIS. Such data analysis approach could help us to come to a better understanding of the obesity and other public health problems in the US. We look forward similar approaches to be applied to other social problems in the US and other countries.

\section{References}

[1] Swinburn, B.A., et al. (2011) The Global Obesity Pandemic: Shaped by Global Drivers and Local Environments. The Lancet, 378, 804-814. https://doi.org/10.1016/S0140-6736(11)60813-1

[2] World Health Organization (2016) Obesity and Overweight. http://www.who.int/mediacentre/factsheets/fs311/en/

[3] Durand, C.P., Andalib, M., Dunton, G.F., Wolch, J. and Pentz, M.A. (2011) A Systematic Review of Built Environment Factors Related to Physical Activity and Obesity Risk: Implications for Smart Growth Urban Planning. Obesity Reviews, 12, e173-e182. https://doi.org/10.1111/j.1467-789X.2010.00826.x

[4] Feng, J., Glass, T.A., Curriero, F.C., Stewart, W.F. and Schwartz, B.S. (2010) The Built Environment and Obesity: A Systematic Review of the Epidemiologic Evidence. Health \& Place, 16, 175-190.

https://doi.org/10.1016/j.healthplace.2009.09.008

[5] Gordon, P., Nelson, M. and Rage, P. (2006) Inequality in the Built Environment Underlies Key Health Disparities in Physical Activity and Obesity. Pediatrics, 117, 417-424.

[6] Sallis, J.F., Floyd, M.F., Rodriguez, D.A. and Saelens, B.E. (2012) The Role of Built Environments in Physical Activity, Obesity, and CVD. Circulation, 125, 729-737. https://doi.org/10.1161/CIRCULATIONAHA.110.969022

[7] Williams, L.M. (2013) Between Health and Place: Understanding the Built Environment. 2013, Wellesley Institute.

[8] Rundle, A., et al. (2009) Neighborhood Food Environment and Walkability Predice Obesity in New York City. Environmental Health Perspectives, 117, 442-447. https://doi.org/10.1289/ehp.11590

[9] Xu, Y. and Wang, F. (2015) Built Environment and Obesity by Urbanicity in the US. Health \& Place, 34, 19-29. https://doi.org/10.1016/j.healthplace.2015.03.010

[10] Saelens, B.E. and Handy, S.L. (2008) Built Environment Correlates of Walking: A Review. Medicine and Science in Sports and Exercise, 40, S550-S566. https://doi.org/10.1249/MSS.0b013e31817c67a4

[11] Sallis, J.F. and Glanz, K. (2009) Physical Activity and Food Environments: Solutions to the Obesity Epidemic. Milbank Q, 87, 123-154. https://doi.org/10.1111/j.1468-0009.2009.00550.x 
[12] Dyck, D.V., et al. (2010) Neighborhood Walkability and Sedentary Time in Belgian Adults. American Journal of Preventive Medicine, 39, 25-32.

[13] Carr, L.J., Dunsiger, S.I. and Marcus, B.H. (2011) Validation of Walk Score for Estimating Access to Walkable Amenities. British Journal of Sports Medicine, 45, 1144-1148. https://doi.org/10.1136/bjsm.2009.069609

[14] Turrell, G., Haynes, M., Wilson, L.-A. and Giles-Corti, B. (2012) Can the Built Environment Reduce Health Inequalities? A Study of Neighbourhood Socioeconomic Disadvantage and Walking for Transport. Health \& Place, 19, 89-98.

[15] Block, J.P., Christakis, N.A., O’Malley, J. and Subramanian, S.V. (2011) Proximity to Food Establishments and Body Mass Index in the Framingham Heart Study Offspring Cohort Over 30 Years. American Journal of Epidemiology, 174, 1108-1114. https://doi.org/10.1093/aje/kwr244

[16] Rahman, T., Cushing, R.A. and Jackson, R.J. (2011) Contributions of Built Environment to Childhood Obesity. Mount Sinai Journal of Medicine, 78, 49-57. https://doi.org/10.1002/msj.20235

[17] Klesges, R.C., Eck, L.H., Hanson, C.L., Haddock, C.K. and Klesges, L.M. (1990) Effects of Obesity, Social Interactions, and Physical Environment on Physical Activity in Preschoolers. Health Phychology, 9, 435-449. https://doi.org/10.1037/0278-6133.9.4.435

[18] Baranowski, T., Thompson, W.O., Durant, R.H., Baranowski, J. and Puhl, J. (1993) Observations on Physical Activity in Physical Locations: Ager Gender, Ethnicity, and Month Effects. Research Quarterly for Exercise and Sport, 64, 127-133. https://doi.org/10.1080/02701367.1993.10608789

[19] Oja, L. and Jürimäe, T. (2002) Physical Activity, Motor Ability, and School Readiness of 6-yr-old Children. Perceptual and Motor Skills, 95, 407-415.

[20] Burdette, H.L. and Whitaker, R.C. (2004) Neighborhood Playgrounds, Fast Food Restaurants, and Crime: Relationships to Overweight in Low-Income Preschool Children. Preventative Medicine, 38, 57-63.

[21] Tucker, T. and Gilliland, J. (2007) The Effect of Season and Weather on Physical Activity: A Systematic Review. Public Health, 121, 909-922.

[22] Hill, J.O. and Melanson, E.L. (1999) Overview of the Determinants of Overweight and Obesity: Current Evidence and Research Issues. Medicine \& Science in Sports \& Exercise, 31, S515-S521. https://doi.org/10.1097/00005768-199911001-00005

[23] Von Hippel, P. and Benson, R. (2014) Obesity and the Natural Environment across US Counties. American Journal of Public Health, 104, 1287-1293. https://doi.org/10.2105/AJPH.2013.301838

[24] Molnar, B., Gortmaker, S.L., Bull, F. and Buka, S. (2004) Unsafe to Play? Neighborhood Disorder and Lack of Safety Predict Reduced Physical Activity among Urban Children and Adolescents. American Journal of Health Promotion, 18, 378-386. https://doi.org/10.4278/0890-1171-18.5.378

[25] Cecil-Karb, R. and Grogan-Kaylor, A. (2009) Childhood Body Mass Index in Community Context: Neighborhood Safety, Television Viewing, and Growth Trajectories of BMI. Health Social Work, 34, 169-177. https://doi.org/10.1093/hsw/34.3.169

[26] Mokhtarian, P.L. and Cao, X. (2008) Examining the Impacts of Residential Self-Selection on Travel Behavior: A Focus on Methodologies. Transportation Research Part B, 42, 204-228.

[27] Gose, M., et al. (2013) Longitudinal Influences of Neighbourhood Built and Social Environment on Children's Weight Status. International Journal of Environmental 
Research and Public Health, 10, 5083-5096. https://doi.org/10.3390/ijerph10105083

[28] Knuiman, M.W., et al. (2014) A Longitudinal Analysis of the Influence of the Neighborhood Built Environment on Walking for Transportation. American Journal of Epidemiology, 180, 453-461. https://doi.org/10.1093/aje/kwu171

[29] Sui, G., Oreskovic, N.M. and Lin, H. (2014) How Do Changes to the Built Environment Influence Walking Behaviors? A Longitudinal Study within a University Campus in Hong Kong. International Journal of Health Geographics, 13, 1-10.

[30] National Center for Health Statistics (2015) Adult Physical Activity Information. Questionnaires, Datasets, and Related Documentation. https://www.cdc.gov/nchs/nhis/physical_activity/pa_comparison.htm

[31] Behavioral Risk Factor Surveillance System (2013) BRFSS 2011 Survey Data and Documentation.

[32] United States Census Bureau (2016) Small Area Income and Poverty Estimates.

[33] Parker, T. (2016) County-Level Data Sets. Economic Research Service: United States Department of Agriculture.

[34] Wang, F., Wen, M. and Xu, Y. (2013) Population-Adjusted Street Connectivity, Urbanicity and Risk of Obesity in the U.S. Applied Geography, 41, 1-14.

[35] TeleMart (2016) Tele Atlas Data for Spatial Technology. ESRI ArcMap Users. http://www.tele-mart.com/esri.php\#

[36] Walk Score Professional (2016) Walk Score API. https://www.redfin.com/how-walk-score-works

[37] Walk Score (2016) Walk Score Methodology. https://www.redfin.com/how-walk-score-works

[38] Wang, F. and Luo, W. (2005) Assessing Spatial and Nonspatial Factors for Healthcare Access: Towards an Integrated Approach to Defining Health Professional Shortage Areas. Health \& Place, 11, 131-146.

[39] Xu, Y. and Wang, L. (2015) GIS-Based Analysis of Obesity and the Built Environment in the US. Cartography and Geographic Information Science, 42, 9-21. https://doi.org/10.1080/15230406.2014.965748

[40] Xu, Y., Wen, M. and Wang, F. (2015) Multilevel Built Environment Features and Individual Odds of Overweight and Obesity in Utah. Applied Geography, 60, 197-203.

[41] Lamichhane, A.P., et al. (2013) Spatial Patterning of Supermarkets and Fast Food Outlets with Respect to Neighborhood Characteristics. Health \& Place, 23, 157-164.

[42] Maddock, J. (2004) The Relationship between Obesity and the Prevalence of Fast Food Restaurants: State-Level Analysis. American Journal of Health Promotion, 19, 137-143. https://doi.org/10.4278/0890-1171-19.2.137

[43] Lopez, R.P. and Hyness, H.P. (2006) Obesity, Physical Activity, and the Urban Environment: Public Health Research Needs. Environmental Health, 5, 25.

[44] Weir, L.A., Etelson, D. and Brand, D.A. (2006) Parents' Perceptions of Neighborhood Safety and Children's Physical Activity. Preventive Medicine, 43, 212-217.

[45] Centers for Disease Control and Prevention (2015) NCHS Urban-Rural Classification Scheme for Counties.

[46] Ingram, D.D. and Franco, S.J. (2013) NCHS Urban-Rural Classification Scheme for Counties. National Center for Health Statistics, Vital Health Stat.

[47] Casagrande, S.S., Gittelsohn, J., Zonderman, A.B., Evans, M.K. and Gary-Webb, T.L. (2011) Association of Walkability with Obesity in Baltimore City, Maryland. 
American Journal of Public Health, 101, S318-S324.

https://doi.org/10.2105/AJPH.2009.187492

[48] Frank, L.D., Andresen, M.A. and Schmid, T.L. (2004) Obesity Relationships with Community Design, Physical Activity, and Time Spent in Cars. American Journal of Preventive Medicine, 27, 87-96.

Submit or recommend next manuscript to SCIRP and we will provide best service for you:

Accepting pre-submission inquiries through Email, Facebook, LinkedIn, Twitter, etc. A wide selection of journals (inclusive of 9 subjects, more than 200 journals) Providing 24-hour high-quality service

User-friendly online submission system Fair and swift peer-review system Efficient typesetting and proofreading procedure Display of the result of downloads and visits, as well as the number of cited articles Maximum dissemination of your research work

Submit your manuscript at: http://papersubmission.scirp.org/ Or contact gep@scirp.org 